\title{
Search for extended gamma-ray halos around AGN with VERITAS
}

\author{
Elisa Pueschel* \\ University College Dublin, Dublin, Ireland \\ on behalf of the VERITAS Collaboration \\ E-mail: elisa.puescheleucd.ie
}

\begin{abstract}
VERITAS, consisting of four $12 \mathrm{~m}$ telescopes designed to detect very high energy (VHE, E $>100$ $\mathrm{GeV}$ ) gamma rays, is one of the most sensitive of the currently operating imaging atmosphericCherenkov telescope arrays. We present the latest VERITAS results on the search for extended gamma-ray halo emission around active galactic nuclei (AGN). Extended halos are predicted to form when multi-TeV VHE gamma rays from AGN interact with extragalactic background light (EBL) photons to produce electron-positron pairs, which then interact with cosmic microwave background (CMB) photons via inverse-Compton scattering to produce lower-energy gamma rays. Depending on the strength of the magnetic fields encountered by the electron-positron pairs before they inverse-Compton scatter, a diffuse halo may form around the beamed gamma rays from the AGN. The search for VHE emission from such haloes therefore probes the strength of the extragalactic magnetic field, as well as the density of the EBL.
\end{abstract}

Science with the New Generation of High Energy Gamma-ray experiments, 10th Workshop - Scineghe2014 04-06 June 2014

Lisbon - Portugal

\footnotetext{
* Speaker.
} 


\section{Introduction}

The strength of the extragalactic magnetic field (EGMF) is of fundamental physical importance, and may provide clues into its generation in the early Universe [1]. The strength of the EGMF is only weakly constrained, with an upper limit of $10^{-9} \mathrm{G}$ and a lower limit of $10^{-20.5} \mathrm{G}$ [2]. Several methods probe the magnetic field strength, each of them sensitive to a different range of magnitudes. The search for gamma-ray pair halos around active galactic nuclei (AGN) probes magnetic field strengths between $10^{-12} \mathrm{G}$ and $10^{-7} \mathrm{G}$ [3]. Previous halo search have been performed by MAGIC, Fermi-LAT, VERITAS and H.E.S.S. [4, 5, 6, 7].

An electromagnetic cascade inevitably develops when multi-TeV gamma rays from active galactic nuclei pass through low-energy photon fields. The energetic gamma rays initially scatter off low-energy extragalactic background light (EBL) photons to produce electron-positron pairs. The electrons and positrons then up-scatter low-energy photons from the cosmic microwave background (CMB) to higher energies via inverse Compton scattering. Depending on the energies, the secondary emission will be observable with imaging atmospheric Cherenkov telescopes (IACTs), in addition to the beamed primary emission. The presence of a non-zero magnetic field will affect the development of the electromagnetic cascade, as the electron-positron pairs will be deflected by the EGMF before inverse-Compton scattering. For weak fields $\left(B<10^{-14} \mathrm{G}\right)$, a magneticallybroadened cascade is produced, as the secondary emission travels roughly concurrently with the beamed emission, but over a slightly longer path length [8]. Stronger fields $\left(10^{-12}-10^{-7} \mathrm{G}\right)$ isotropize the electron-position pairs, which will collect in the vicinity of the AGN. The secondary gamma-ray emission produces a symmetric GeV "halo" around the AGN [3]. For intermediate field strengths $\left(10^{-13} \mathrm{G}\right)$, significant isotropization will occur, rendering the magnetically-broadened cascade component dim, but the halo component will be too large and diffuse to be detectable.

If it is sufficiently bright in comparison to the beamed emission, the halo will be detectable with an IACT from the source's observed angular profile. IACTs define a parameter $\theta^{2}$ which characterizes the angular profile, defined as the angular distance between the air shower's arrival direction and source's estimated location. For a point source, the $\theta^{2}$ distribution is sharply peaked at zero, with a width dependent on the telescope array's point spread function (PSF). A flat background from cosmic ray-induced showers with no specified arrival direction will appear under the signal peak. Thus, it is expected that extended emission could be observed as a broadening in the angular profile. The expected distribution in this case would be the sum of a point-like distribution from the beamed emission and a broader angular profile from the halo.

Angular profiles due to pair halos have been studied extensively via simulation [3]. For magnetic field strengths sufficient to produce halos, the profile is insensitive to the exact magnetic field strength. The profile is, however, sensitive to source redshift, the injection energy of the initial photons from the AGN, and the energy and spectral index of the secondary gamma rays. The choice of EBL also affects the predicted profile. Halos are predicted to be more compact for more distant sources and for higher energy initial photons.

\section{The VERITAS instrument}

VERITAS is an array of four 12 meter IACTs [9]. It is located at Fred Lawrence Whipple 


\begin{tabular}{cccc} 
& $z$ & $\Phi(\mathrm{CU})$ & $\sigma$ \\
\hline \hline Mrk 421 & 0.031 & 0.3 & 81.6 \\
1ES 2344+514 & 0.044 & 0.11 & 9.6 \\
1ES 1959+650 & 0.048 & 0.64 & 14.4 \\
1ES 0229+200 & 0.14 & 0.018 & 11.1 \\
1ES 1218+304 & 0.182 & 0.08 & 37.8 \\
PG 1553+113 & 0.5 & 0.034 & 41.4 \\
\hline \hline
\end{tabular}

Table 1: AGNs analyzed, with their redshift, flux in Crab units, and detection significance for the dataset used in this measurement.

Observatory in southern Arizona, and has been in operation since 2007. VERITAS is sensitive to very high energy gamma rays in an energy range from $100 \mathrm{GeV}$ to greater than $30 \mathrm{TeV}$. Following a major upgrade in 2012, VERITAS has extended sensitivity at lower energies. VERITAS has an energy resolution of $15 \%$ and an angular resolution of $0.1^{\circ}$ at $1 \mathrm{TeV}$. The field of view is $3.5^{\circ}$, and peak effective area is $100,000 \mathrm{~m}^{2}$ above $\sim 600 \mathrm{GeV}$. The instrument is capable of detecting a point source of $1 \%$ Crab flux at the $5 \sigma$ level in less than 30 hours.

\subsection{VERITAS pointing monitor}

Telescope pointing is a major potential systematic in this measurement. Accurate pointing is necessary to characterize the PSF. VERITAS employs the VERITAS Pointing Monitor (VPM) to measure the pointing as observations are taken. Each telescope is equipped with a CCD camera for optical monitoring of the pointing. Images are taken every 2 seconds, ensuring real-time information. The estimated systematic uncertainty on the pointing is $50^{\prime \prime}$, which is negligible in comparison to the telescope array's angular resolution.

\section{Source selection}

The characteristics of the source strongly affects the projected sensitivity to extended emission. As described in the introduction, the angular size of the halo is inversely proportional to the source redshift [3]. Ideal candidates will be located at a distance where the extended emission is neither so compact that it is indistinguishable from the beamed emission, nor so broad that it is indistinguishable from the cosmic-ray background; redshifts of $z \approx 0.1-0.24$ (few hundred megaparasecs to one giga-parasec) are optimal. Hard-spectrum sources with emission detected above 1 $\mathrm{TeV}$ are favored for searches for cascade emission in around $100 \mathrm{GeV}$. Soft-spectrum blazars will produce cascade emission that largely falls below the VERITAS energy threshold.

The six sources selected are listed in Table 1 . They cover a broad range of redshifts, including the range considered to be most favorable. The sources were also selected based on the significance of their detection, with the goal of minimizing statistical uncertainties in the resulting constraints on extended emission.

Both Markarian 421 and 1ES1959+650 experienced flares during the observation periods considered here. During a flare, the number of gamma rays from extended emission will be negligible 

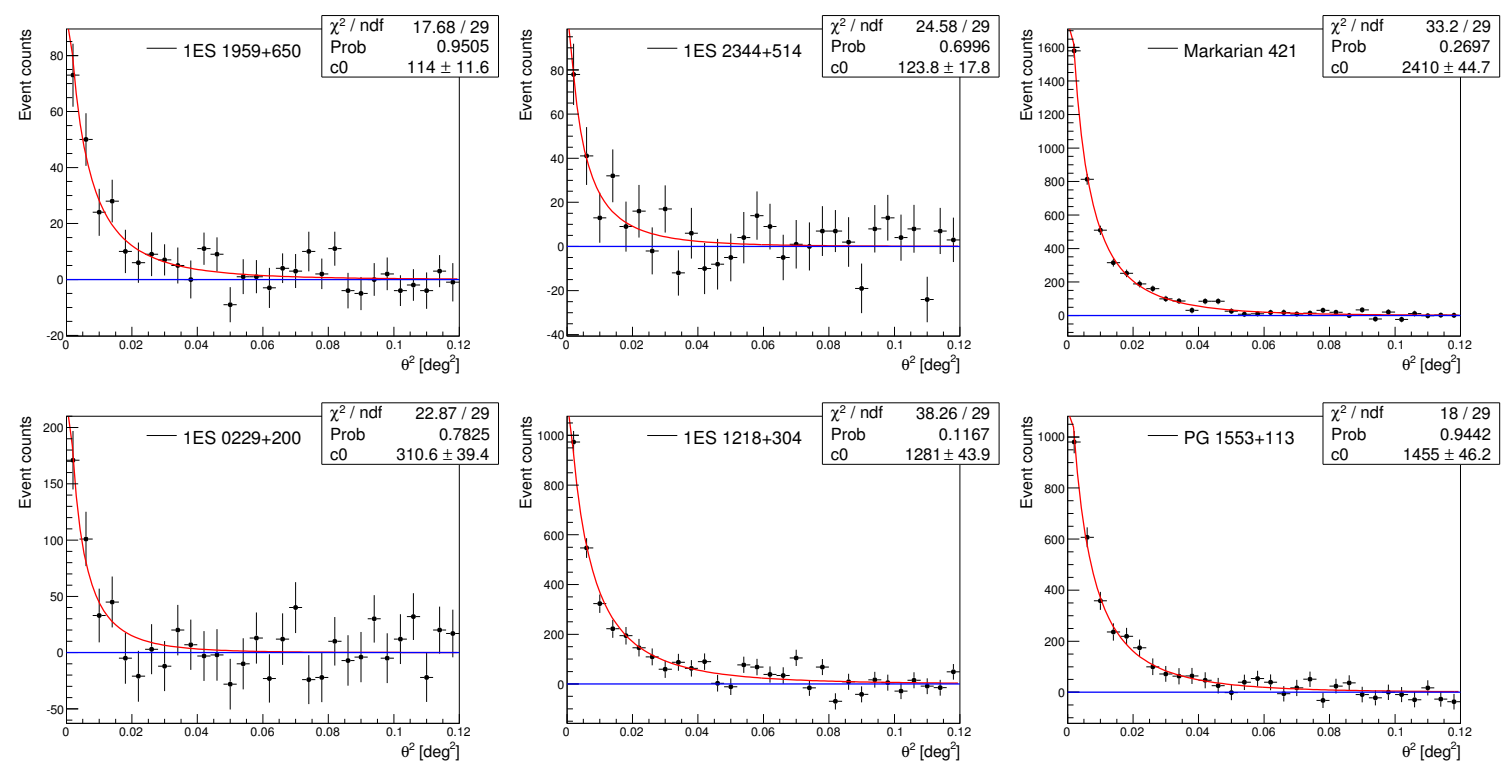

Figure 1: Data angular profiles for the six AGN. The data are fit with a sech function with the width fixed to the value derived from simulation, with the exception of Markarian 421, whose expected width is derived from the April 2013 flare data.

in comparison to the number from beamed emission, and the source will appear point-like. Thus, the flare data are inappropriate for a search for extended emission and are not included.

\section{Analysis strategy}

The VERITAS PSF varies with the zenith and azimuth of the observed source, and with the source's energy spectrum. The sources considered in this analysis were observed at a range of zenith and azimuth angles. Though the distributions overlap for some sources, it is inadequate to use a single value of the PSF for all six sources. Monte Carlo simulation is used to generate an expected PSF for each source that accounts for the differences in the observing conditions. Simulated events with the appropriate zenith and azimuth range were selected for each source, and the reconstructed energy distribution in simulation was re-weighted to match the energy distribution of the data. The exception to this procedure was Markarian 421, for which simulation was not available with the appropriate zenith and azimuth range. However, Markarian 421 experienced a bright flare in April 2013. As stated previously, a flaring source is expected to be point-like and the width of its $\theta^{2}$ distribution will reflect the PSF. The data from this period were used to derive the expected PSF for Markarian 421.

To derive the PSFs, each simulated source (or in the case of Markarian 421, flare data) was fit with a hyperbolic secant function, resulting in a width $\sigma_{S I M}$ that describes the PSF for a point source. The $\theta^{2}$ distribution in data for each source was then also fit with a hyperbolic secant, with the width fixed to $\sigma_{S I M}$. In all cases, $\sigma_{S I M}$ adequately describes the observed width of the $\theta^{2}$ distribution in data. The fit results are shown in Figure 1. The fit probabilities are comparable to probabilities from fits that allow the hyperbolic secant's width to float. 

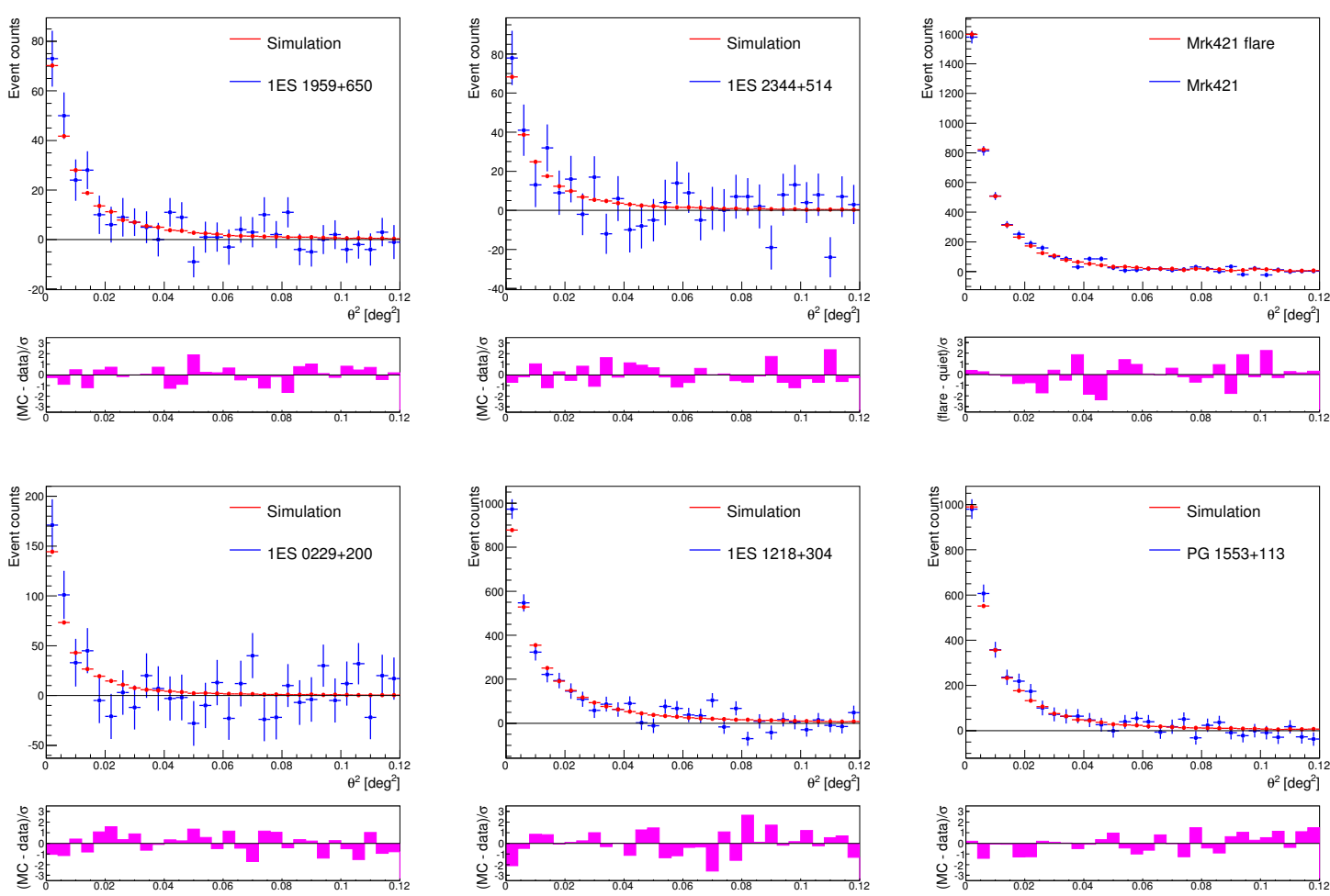

Figure 2: Overlay of data angular profiles with simulated angular profiles.

\section{Results and Discussion}

No deviation is observed between the observed $\theta^{2}$ distributions and the predictions for a point source. With no evidence for the detection of extended emission, upper limits can be set on the halo flux. Preliminary model-independent limits were set based on the data-simulation comparisons shown in Fig. 2. The data and Monte Carlo distributions are in good agreement. The bulk of the beamed emission is expected to fall in the range $\theta^{2}=0.0-0.1$, thus an excess due to extended emission was calculated:

$$
\text { excess }=\int_{0.01}^{0.12} \theta_{\text {Source }}^{2}-\int_{0.01}^{0.12} \theta_{\text {SIM }}^{2}
$$

Upper limits on the flux from extended emission are then derived using the method of Helene [10]. The 95\% confidence level upper limits are shown in Table 2.

As the electron-position density around an AGN and the resulting halo brightness depend on the blazar's activity over the course of its lifetime, it is not possible to constrain the field strengths in the range necessary to produce a halo based on non-detection. However, non-detection of extended emission constrains fields of order $10^{-15} \mathrm{G}-10^{-14} \mathrm{G}$. For these weaker fields, the $\mathrm{e}^{+} / \mathrm{e}^{-}$pairs are not isotropized and are basically concurrent with the beamed emission, and thus the predicted extended emission is independent of the blazar's past activity. 


\begin{tabular}{cc} 
& $95 \%$ CL Upper Limit (\% Crab Flux) \\
\hline \hline Mrk 421 & $4.0 \%$ \\
1ES 2344+514 & $2.1 \%$ \\
1ES $1959+650$ & $1.9 \%$ \\
1ES $0229+200$ & $0.9 \%$ \\
1ES $1218+304$ & $0.8 \%$ \\
PG $1553+113$ & $0.8 \%$ \\
\hline \hline
\end{tabular}

Table 2: Preliminary model independent upper limits on flux from extended emission, in Crab units.

\section{Conclusions}

A search for pair halos around AGN was completed using deep VERITAS observations of six AGN at a range of redshifts. The angular profiles for all the AGN were consistent with emission by a point source, with no evidence for extended emission from a halo. Preliminary modelindependent limits of a few percent Crab flux were set on the possible contribution from extended emission. The sources studied are included in the VERITAS long term monitoring plan, and thus an updated result including more data and analysis improvements will be produced in the future.

\section{Acknowledgments}

This research is supported by grants from the U.S. Department of Energy Office of Science, the U.S. National Science Foundation and the Smithsonian Institution, by NSERC in Canada, by Science Foundation Ireland (SFI 10/RFP/AST2748) and by STFC in the U.K. We acknowledge the excellent work of the technical support staff at the Fred Lawrence Whipple Observatory and at the collaborating institutions in the construction and operation of the instrument. The VERITAS Collaboration is grateful to Trevor Weekes for his seminal contributions and leadership in the field of VHE gamma-ray astrophysics, which made this study possible. Funding for this research was provided by the Irish Research Council's Government of Ireland Postdoctoral Research Fellowship.

\section{References}

[1] R. Durrer and A. Neronov. The Astronomy and Astrophysics Review 21 (2013)

[2] K. Takahashi et al. Astrophys. J., 771:L42 (2013)

[3] A. Eungwanichayapant and F. Aharonian. Inter. J. of Modern Physics D, 18(6), 911-927 (2009)

[4] MAGIC collaboration. Astronomy \& Astrophysics 524, A77 (2010)

[5] Fermi-LAT collaboration. Astrophys. J., 765:54 (2013)

[6] M. Fernandez Alonso. BAAA (Boletín de la Asociación Argentina de Astronomía), 56 (2013)

[7] H.E.S.S. collaboration. Astronomy \& Astrophysics, 562:A145 (2014)

[8] A. Elyiv et al. Phys. Rev. D 80:023010 (2009)

[9] VERITAS collaboration. Astroparticle Physics 25, 391-401 (2006)

[10] O. Helene. Nuclear Instruments and Methods in Physics Research, 212, 319 (1983) 